\title{
Effect of Polyimide Layers on the Permittivity Tuning Range of Liquid Crystals
}

\author{
Pouria Yaghmaee $^{\# 1}$, Thomas Kaufmann ${ }^{\# 2}$, Bevan Bates ${ }^{\# *^{3}}$, Christophe Fumeaux ${ }^{\# 4}$ \\ ${ }^{\#}$ School of Electrical and Electronic Engineering University of Adelaide, Adelaide, SA 5000, Australia \\ 1pouria@eleceng.adelaide.edu.au \\ 2 thomaska@eleceng.adelaide.edu.au \\ 4cfumeaux@eleceng.adelaide.edu.au \\ * Defence Science and Technology Organisation (DSTO), Edinburgh, SA 5111, Australia \\ 3 bevan.bates@dsto.defence.gov.au
}

\begin{abstract}
Liquid crystals have potential applications in the design of tunable microwave devices. Polyimide films are commonly incorporated in designs to achieve reproducible molecule alignment in the unbiased state. In this investigation two separate resonator elements are designed and investigated: One is fabricated with polyimide layers and one without. Experimental results obtained with a patch resonator illustrate that about half of the maximum tuning range is achieved without polyimide layers. Since substrates able to sustain a temperature range of $180-300{ }^{\circ} \mathrm{C}$ are required for the polyimide film curing process, avoiding polyimide enables using even lower heat resistant substrates for various tunable microwave applications and can lead to faster fabrication processes.
\end{abstract}

Keywords-component; tunable devices, liquid crystal (LC), polyimide film, tunable resonator.

\section{INTRODUCTION}

Due to various design challenges such as frequency variations and dynamic changes in environmental conditions considerable research efforts have been invested toward tunable microwave devices. In order to design a frequency tunable device, five main methods can be used [1]: Mechanical change of the physical dimensions, integration of microwave diodes, integration of micro-electro-mechanical systems (MEMS) switches, change of the substrate permittivity and integration of voltage controlled membranes. Frequency variation in microwave devices has also been demonstrated through integration of voltage-controlled changes in the substrate permittivity.

Examples of this last method were described in [2], for barium strontium titanate and in [3] for ferroelectric materials. Liquid crystals (LC) have also recently been discussed as substrates with variable permittivity for designing tunable microwave devices, mainly at frequencies above $10 \mathrm{GHz}$. Examples of such tunable microwave devices can be found in [4-6] for antennas and reflectarrays, in [7] for phase shifters and in [8] for delay lines.

\section{LIQUID CRYSTAL PROPERTIES}

The strong motivating advantages of LC materials in the view of usage in tunable microwave devices are:

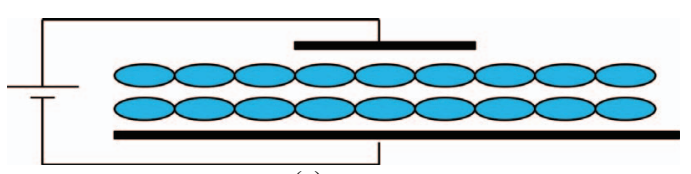

(a)

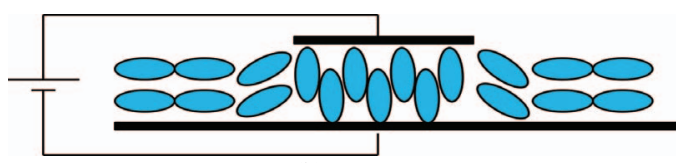

(b)

Fig.1 Illustration of the change in the LC molecules orientation as bias voltage is applied (a) for when $V_{b}=0 \mathrm{~V}$ is applied (b) for when $V_{b} \gg V_{t h}$ is applied.

- Continuous tuning of their dielectric characteristics,

- Integration in printed technology and in flexible electronic devices,

- Low bias voltage requirements, and

- Wide commercial availability in the electronic industry.

The liquid crystal nematic phase is the most common phase of liquid crystals and is characterized by the orientation of rod-shaped molecules resulting in a highly anisotropic material characteristics at microwave frequency. By applying a bias voltage $V_{b}$ across the LC cell, the molecule orientation can be changed from perpendicular to parallel through alignment of the molecules along the electric field. The electric field required to change the direction of the LC molecules from perpendicular to parallel is known as Fredericks Threshold. This corresponds to a voltage $V_{t h}$ typically around 15 to $30 \mathrm{~V}$ depending on the LC type and for cavity thicknesses typically used for microwave devices. The dielectric anisotropy, $\Delta \varepsilon_{r}$ can be written as:

$$
\Delta \varepsilon_{r}=\varepsilon_{r \prime \|}-\varepsilon_{r{ }^{\prime} \perp}
$$

In this equation, $\varepsilon_{r, \perp}=\varepsilon\left(V_{b}=0 \mathrm{~V}\right)$ is the perpendicular permittivity for zero voltage and $\varepsilon_{r^{\prime} \|}=\varepsilon\left(V_{b} \gg V_{t h}\right)$ is the parallel permittivity at maximum bias voltage $V_{b}$ (see Fig.1). In practical designs both the top and bottom layers of the cells need to be spin-coated with a thin layer of polyimide films to help in the alignment of the LC molecules in the unbiased 
state. In the following, the influence of these polyimide films on tuning range is estimated.

\section{DESIGN IMPLEMENTATION}

\section{A. Resonator Structure}

The designed resonator element consists of three layers: a) a resonant metal patch in the centre of feeding microstrip lines on a flipped substrate (top layer), b) a dielectric substrate with a cut-out cavity (middle/spacer layer) and c) a metal ground plane (bottom layer) (Fig. 2).

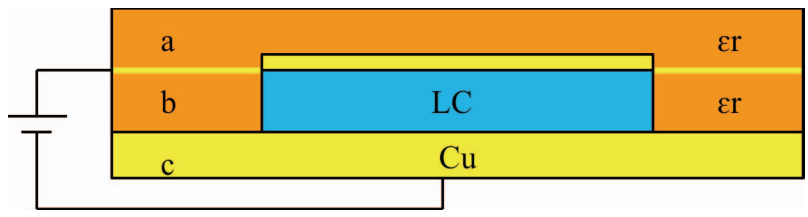

Fig. 2 Cut view of the designed tunable resonator filled with LC, showing all three layers.

\section{B. Resonator Element Fabrication}

On the top layer, a $28 \mathrm{~mm} \times 28 \mathrm{~mm}$ patch and two connecting $50 \Omega$ microstrip lines are patterned on a Rogers $\mathrm{RT} /$ Duroid 5880 substrate with a relative permittivity of $\varepsilon_{r}=$ 2.2. The spacer layer is designed using the same Duroid material with a thickness of $0.5 \mathrm{~mm}$. A square slot is cut in the centre of this spacer layer to form the cavity for the LC.

A square cavity with identical dimensions and a depth of $0.5 \mathrm{~mm}$ is also cut in the metal bottom layer. This cavity is fitted with a Rogers TMM3 substrate $\left(\varepsilon_{r}=3.27\right)$ with a 0.5 $\mathrm{mm}$ thickness, which creates a surface for the polyimide layers to be included at the lower boundary of the cavity (Fig. $3)$. The two microstrip lines on both sides of the top layer patch are connected to SMA connectors to enable measurements of the transmission and reflection coefficients.

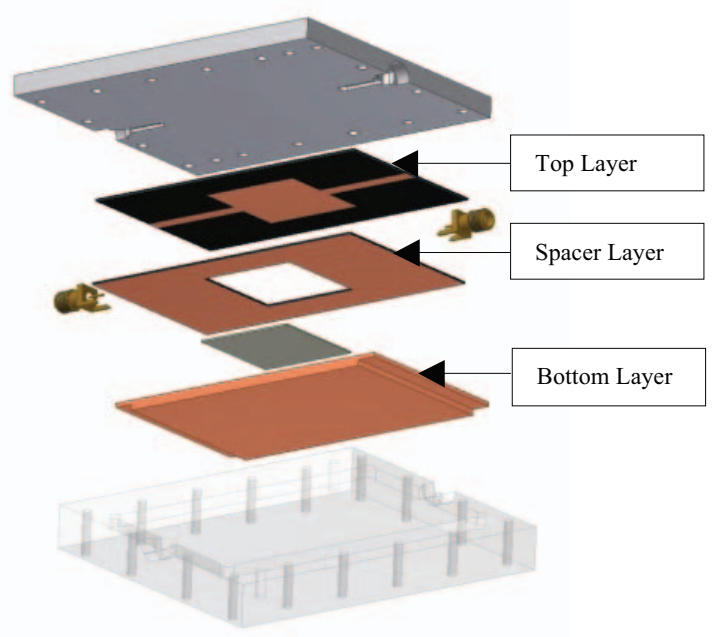

Fig. 3 Side view of the designed resonator, showing all layers and the top and bottom covers.
TABLE I. PROPERTIES OF K15 LIQUID CRYSTAL AT $1 \mathrm{GHz}$

\begin{tabular}{|c|c|c|c|}
\hline Permittivity & $\boldsymbol{\varepsilon}$ & Loss $\tan \boldsymbol{\delta}$ & $\boldsymbol{\Delta} \boldsymbol{\varepsilon}$ \\
\cline { 1 - 2 }$\varepsilon_{\perp}$ & 2.72 & \multirow{2}{*}{$3.10^{-2}$} & 0.18 \\
\hline$\varepsilon_{\|}$ & 2.90 & & \\
\hline
\end{tabular}

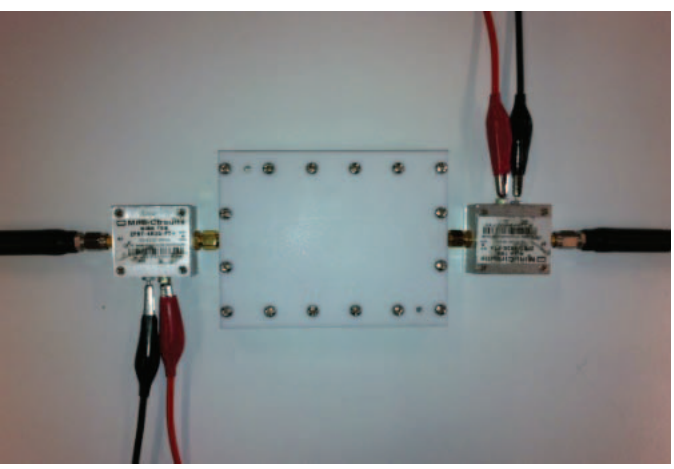

Fig. 4 Packaged resonator element filled with K15-LC and connected to biastees.

The standard liquid crystal K15 [9] was used for filling the cavity of this resonator element. The values of perpendicular and parallel permittivity for this material are presented in Table I. Finally the complete resonator element, as illustrated in Fig. 4, is placed in Teflon top and plexiglass bottom covers to seal the cavity and avoid any liquid leakage.

\section{Simulation Results}

The designed resonator element is simulated in CST Microwave Studio [10] over a microwave frequency range of 2.0-4.5 GHz. For this simulation the relative permittivity values from Table I are used. Simulation results for both $S_{11}$ and $S_{21}$ parameters for $V_{b}=0 \mathrm{~V}\left(\varepsilon_{\perp}=2.72\right)$ and $V_{b}=$ $30 \mathrm{~V}\left(\varepsilon_{\|}=2.9\right)$ are shown in Fig. 5 .

It is predicted from these simulations that when the relative permittivity changes from $\varepsilon_{r}=2.72$ (perpendicular state) to $\varepsilon_{r}=3.28$ (parallel state), the resonant frequency will shift from $3.28 \mathrm{GHz}$ to $3.14 \mathrm{GHz}$, providing approximately $4.4 \%$ of frequency tuning. The bandwidth for $\mathrm{S}_{11}<-10 \mathrm{~dB}$ is $250 \mathrm{MHz}$ for the unbiased state and $225 \mathrm{MHz}$ for the biased state.

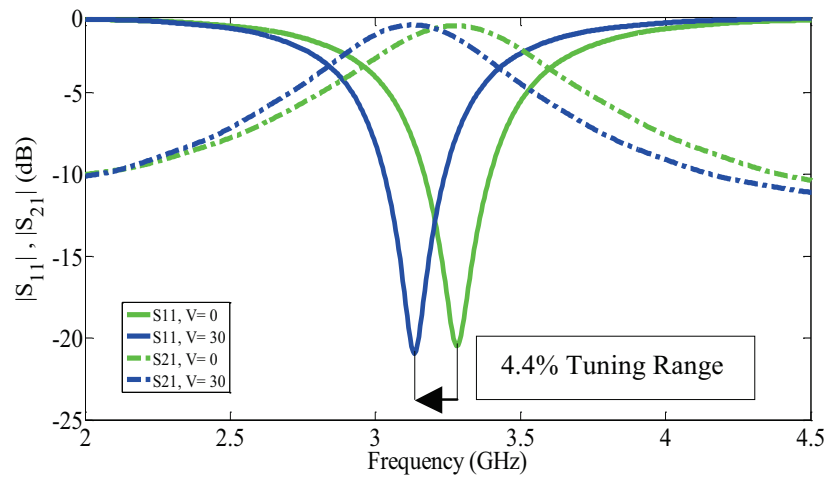

Fig. 5 CST simulation for the resonator element with LC filling in biased and unbiased state. 
The top layer is simulated with a relative permittivity of $\varepsilon_{r}$ $=2.2$, permeability of $\mu_{r}=1$ and a loss tangent $\tan \delta=$ $9 \cdot 10^{-4}$, corresponding to RT/Duroid 5880 substrate values. The bottom layer is simulated with a relative permittivity of $\varepsilon_{r}$ $=3.27$, permeability of $\mu_{r}=1$ and $\tan \delta=2 \cdot 10^{-3}$, corresponding to the TMM3 substrate. The S11 parameters at resonance for both cases are around $-21 \mathrm{~dB}$ and the maximum S21 parameters are better than $-1 \mathrm{~dB}$.

\section{MEASUREMENT RESULTS}

The alignment of the LC molecules in unbiased state is achieved through coating of the copper patch and the TMM3 bottom layer with a thin layer of polyimide film. A layer thickness of about 3-4 $\mu \mathrm{m}$ is produced using a spinner machine. These layers are then placed in an oven for 1 hour at a temperature of $300{ }^{\circ} \mathrm{C}$ for a curing process to take place. The curing temperature might vary between $180-300{ }^{\circ} \mathrm{C}$ depending on the type of polyimide used and available time. The curing process should take place slowly, since baking the substrate in the oven at constant temperature of $300{ }^{\circ} \mathrm{C}$ might have an unpredictable effect on the electromagnetic characteristics of some standards RF substrates.

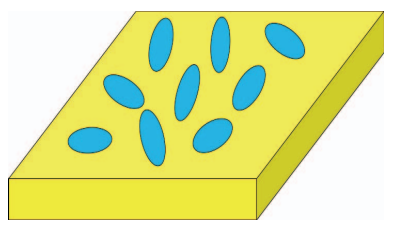

(a)

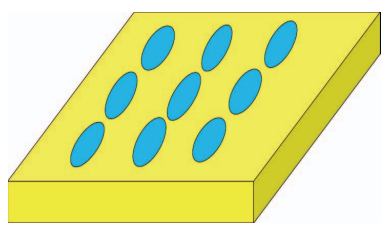

(b)

Fig. 6 (a) Random orientation of the LC molecules without polyimide layer (b) Aligned LC molecules after introduction of a velvet-rubbed polyimide layer.

Finally these polyimide layers are mechanically rubbed using a velvet cloth. The rubbing creates microscopic grooves in the polyimide layer which provides a preferred alignment for the LC molecules along the rubbing direction. (See Fig. 6 (a) and (b)). To quantify the effect of the polyimide films, a comparison is made between a device without polyimide layers and the same design including polyimide layers. The measurement results for biased and unbiased cases are then compared with the CST simulation results.

\section{A. Measurements without Polyimide Layers}

In the first measurement (Fig. 7) the bottom and the top layers are placed in the resonator element without any polyimide coating around the LC cavity. A bias voltage $V_{b}$ is applied between the patch and the ground plane through Mini-

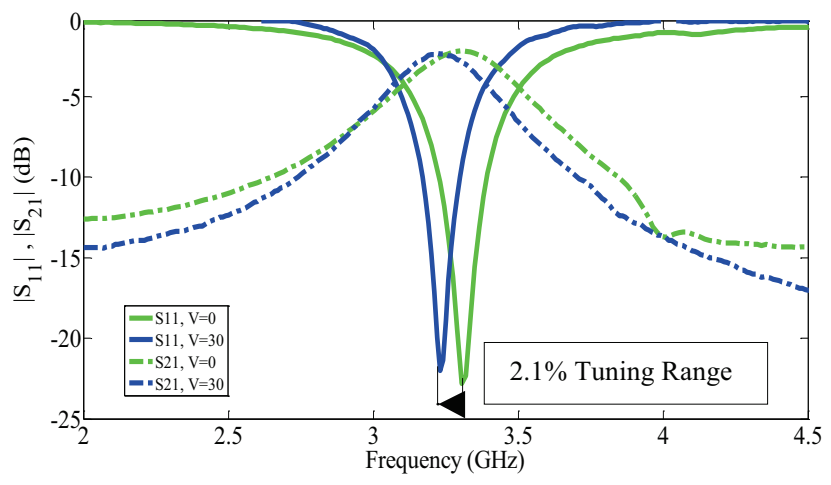

Fig. 7 Measurement results in the absence of polyimide layers.

Circuits wideband bias-tees. The measurement results are shown in Fig. 7, and a comparison between the simulation and measurements in absence of polyimide layers is given in Table II and Fig. 7. The results show that by applying a bias voltage from $0 \mathrm{~V}$ to $30 \mathrm{~V}$, the frequency shifts from $3.30 \mathrm{GHz}$ to $3.23 \mathrm{GHz}$, corresponding to a tuning range of $2.1 \%$. The minimum input reflection coefficients $\left(S_{11}\right)$ for both cases here are around $-23 \mathrm{~dB}$ and transmission coefficients $\left(\mathrm{S}_{21}\right)$ are -1 $\mathrm{dB}$ for simulation and $-2.2 \mathrm{~dB}$ for measurements. The difference between these two values is mainly due to coaxial connector losses.

The results show that even in the absence of the polyimide layer, i.e. with random alignment of LC molecules in unbiased state (Fig. 6 (a)), close to half of the maximum simulated tuning range can be obtained.

\section{A. Measurements with Polyimide Layers}

In the second experiment the bottom (TMM3) and top layer (RT/Duroid 5880) were spin-coated with thin layers of polyimide film and then cured for approximately an hour at $300{ }^{\circ} \mathrm{C}$ (Fig. 8). The surfaces are then mechanically softly rubbed using a velvet cloth.

These layers then form a sandwich cell structure and enclose the K15 liquid in its cavity, with Duroid and metal on top and bottom (Fig. 3). By applying a bias voltage from $V_{b}=0 \mathrm{~V}$ and gradually increasing it to the maximum value of $V_{b}=30 \mathrm{~V}$ the LC molecules change their orientation from parallel to the ground plane to perpendicular. Measurement results in the presence of polyimide layers are presented in Fig. 9 and compared to simulation in Table II.

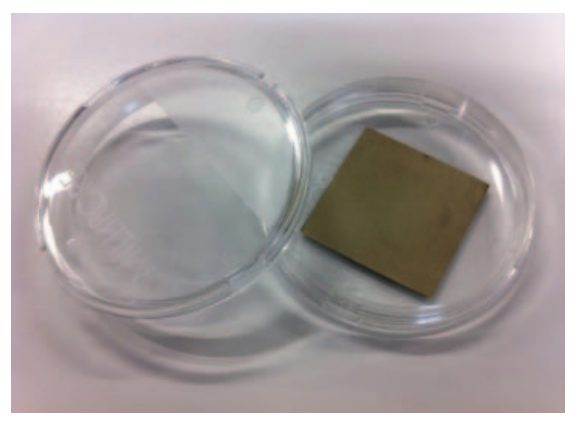

Fig. 8. Spin-coated polyimide surface layer with cured film. 
TABLE II. SIMULATION AND MEASUREMENT COMPARISON FOR THE TUNING RANGE WITHOUT / WITH POLYIMIDE LAYERS INCLUDED

\begin{tabular}{|c|c|c|c|}
\hline $\begin{array}{c}\text { Bias } \\
\text { voltage } \boldsymbol{V}_{\boldsymbol{b}}\end{array}$ & $\begin{array}{c}\text { Simulation } \\
(\mathbf{G H z})\end{array}$ & $\begin{array}{c}\text { Measurement } \\
\text { (GHz) } \\
\text { (without polyimide) }\end{array}$ & $\begin{array}{c}\text { Measurement } \\
\text { (GHz) } \\
\text { (with polyimide) }\end{array}$ \\
\hline $0 \mathrm{~V}$ & 3.28 & 3.30 & 3.30 \\
\hline $30 \mathrm{~V}$ & 3.14 & 3.23 & 3.17 \\
\hline
\end{tabular}

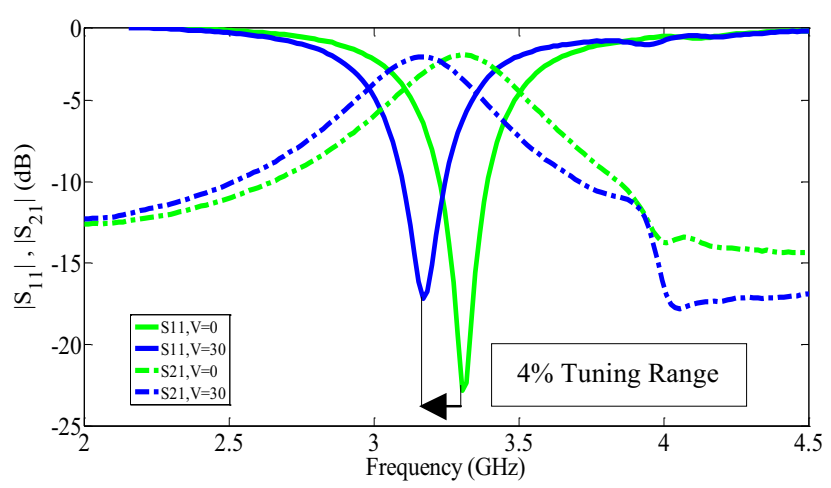

Fig. 9 Measurement results in the presence of polyimide layers.

Fig. 9 shows that by adding polyimide layers to the top and bottom of the cell, i.e. to the surface which are in contact with the K15 LC, a frequency shift of $4 \%$ from $3.30 \mathrm{GHz}$ to $3.17 \mathrm{GHz}$ is achieved. The results also illustrate that the application of polyimide layers and the curing process do not have any effect on the initial centre frequency. The bandwidth remains similar to the previous case at about $175 \mathrm{MHz}$ with no voltage applied and is decreased to $150 \mathrm{MHz}$ when an electric field above $V_{t h}$, i.e. $V_{b}=30 \mathrm{~V}$ is applied.

The drop in the S21 curves around 4.1-4.2 GHz in both cases is explained by the maximum range of the bias-tee used $(4.2 \mathrm{GHz})$. This result shows a satisfactory correspondence with the simulation results.

\section{CONCLUSION}

Two different tunable resonators have been fabricated using standard K15 liquid crystal. A tuning range of $2.1 \%$ is achieved when the boundary layers of the LC cavity are free of polyimide coating and $4 \%$ tuning range is gained when a polyimide layers rubbed with a velvet cloth are applied. Although polyimide layers are common for providing LC molecules alignment in unbiased state, thus increasing the tuning range, this study has quantified the tuning range in absence of these polyimide layers, demonstrating half of the predicted tuning range.
Since the curing process of polyimide layers requires a temperature of $180-300{ }^{\circ} \mathrm{C}$, temperature sensitive substrates could be used nevertheless to bypass the application of polyimide layers, however at the cost of reduced tuning performance. Avoiding this stage of fabrication process would also mean elimination of the curing process which for most polyimide used in RF applications can take between 1 to 3 hours depending on the polyimide and type of substrate used.

\section{ACKNOWLEDGMENT}

The authors wish to acknowledge Mr Igor Switala from the Defence Science and Technology Organisation (DSTO) for providing the polyimide and also $\mathrm{Mr}$ Pavel Simcik and $\mathrm{Mr}$ Brandon Pullen from The University of Adelaide.

\section{REFERENCES}

[1] Petosa, A., "Frequency agile antennas for wireless communications - A survey," 14th International Symposium on Antenna Technology and Applied Electromagnetics \& the American Electromagnetics Conference (ANTEM-AMEREM), pp.1-4, 5-8 July. 2010.

[2] Huff, G.H., Rolando, D.L., Walters, P., McDonald, J., "A Frequency Reconfigurable Dielectric Resonator Antenna Using Colloidal Dispersions," IEEE Antennas and Wireless Propagation Letters, vol. 9, pp. 288-290, 2010.

[3] Lovat, G., Burghignoli, P., Celozzi, S., "A Tunable Ferroelectric Antenna for Fixed-Frequency Scanning Applications," IEEE Antennas and Wireless Propagation Letters, vol. 5, no. 1, pp. 353-356, Dec. 2006.

[4] Gaebler, A., Moessinger, A., Goelden, F., Manabe, A., Goebel, M. Follmann, R., Koether, D., Modes, C., Kipka, A., Deckelmann, M., Rabe, T., Schulz, B., Kuchenbecker, P., Lapanik, A., Mueller, S., Haase, W., Jakoby, R., "Liquid Crystal-Reconfigurable Antenna Concepts for Space Applications at Microwave and Millimeter Waves," International Journal of Antennas and Propagation, Jan. 2009.

[5] Liu, L., Langley, R.J., "Liquid Crystal Tunable Microstrip Patch Antenna," IET Electronic Letters, vol. 44, no. 20, pp. 1179-1180, September 2008 .

[6] Hu, W., Ismail, M.Y., Cahill, R., Gamble, H.S., Dickie, R., Fusco, V.F., Linton, D., Rea, S.P., Grant, N., "Tunable Liquid Crystal Reflectarray Patch Element," IET Electronic Letters, vol. 42, no. 9, pp. 509-511, 2006.

[7] Mueller, S., Goelden, F., Scheele, P., Wittek, M., Hock, C., Jakoby, R., "Passive Phase Shifter for W-Band Applications using Liquid Crystals," 36th European Microwave Conference, 2006. pp. 306-309, 10-15 Sept. 2006.

[8] Kuki, T., Fujikake, H., Nomoto, T., "Microwave variable delay line using dual-frequency switching-mode liquid crystal," IEEE Transactions on Microwave Theory and Techniques, vol. 50, no. 11, pp. 2604-2609, Nov. 2002

[9] Martin, N., Laurent, P., Person, C., Le Roy, M., Perennec, A., Gelin, P., Huret, F., "Influence of design liquid crystal-based devices on the agility capability," IEEE MTT-S International Microwave Symposium Digest, 2005.

[10] Computer Simulation Technology webpage on CST. [Online]. Available: http://www.cst.com 